\title{
O PROCESSAMENTO DE ORAÇÕES RELATIVAS COM E SEM O PRONOME “THAT” POR BRASILEIROS APRENDIZES TARDIOS DE INGLÊS COMO L2 ${ }^{1}$
}

\author{
Alyson Andrade Gonçalves \\ Márcio Martins Leitão ${ }^{2}$
}

\begin{abstract}
The purpose of this study is to investigate the linguistic processing of L2 English by a group of Brazilian native speakers. In order to achieve this goal, we analyze the processing of unambiguous object relative clauses which contain contrasting structures in the two languages studied here, that is, that have two structure options in English (with or without the pronoun "that") but only one in Portuguese (containing "that"). Thus, in an online self-paced reading experiment, we examined the extent to which Brazilian native speakers would show advantages in processing sentences containing the relative pronoun "that", once this structure resembles the one found in Portuguese. Results show that basic, intermediate and advanced learners seem to process object relative clauses more quickly when they present "that" explicitly, which is taken as an indication of the presence of L1 transfer effects in language processing. Such results are discussed within the predictions of the Shallow Structure Hypothesis (Felser et al. 2002).
\end{abstract}

Palavras-chave: processamento lingüístico, aquisição de orações relativas de objeto, inglês como L2, transferência da L1 para L2, Hipótese da Estrutura Rasa (Shallow Structure Hypothesis).

\footnotetext{
1 Trabalho realizado com auxílio do CNPq (Projeto Casadinho - Processo N. 620020/2008-3 e Edital Jovens Pesquisadores - Processo N. 564184/2008-0).

2 Universidade Federal da Paraíba (UFPB).

Organon, Porto Alegre, nº 51, julho-dezembro, 2011, p. 129-144
} 


\section{INTRODUÇÃO}

Nas últimas décadas, muitos fenômenos têm sido estudados em relação ao processamento linguístico de adultos e crianças monolíngues, mas existem ainda poucos trabalhos sobre o processamento linguístico de aprendizes tardios de uma língua estrangeira (LE) ou segunda língua (L2) ${ }^{3}$. Existem estudos que focalizam o aprendizado de L2 em termos de aquisição da linguagem, geralmente analisando a produção de sujeitos aprendizes, entretanto, em relação a como se dá o processamento lingüístico nesses indivíduos, ainda há mais dúvidas do que respostas.

Alguns estudos, como os de Felser e Clahsen (2009), por exemplo, têm mostrado, seja por meio de técnicas psicolinguísticas, seja por técnicas neurolinguísticas, que mesmo indivíduos muito proficientes em uma L2 apresentam diferenças de processamento em comparação com falantes nativos. Em outras palavras, mesmo que esses aprendizes, a "olho nu", possam passar por nativos, quando investigados em termos de processamento, eles mostram que há possíveis distinções ligadas ao desenvolvimento na aquisição da língua materna (doravante, L1) e da L2 que se refletem no processamento linguístico.

Outro aspecto relevante a ser investigado é em que medida existe transferência de estratégias de processamento da L1 para L2, ou seja, quando um determinado fenômeno linguístico é processado diferentemente por nativos da L1 e da L2, será que a estratégia de processamento utilizada na L1 é usada também por aprendizes na L2? E se assim for, em que medida o nível de proficiência na L2 afeta essa transferência?

Com base nessas questões, este estudo visa buscar possíveis respostas ou indícios para compreender um pouco melhor como se dá o processamento linguístico em aprendizes de L2, particularmente, em aprendizes de inglês como L2, tendo como L1 o português brasileiro.

O foco central de nossa investigação é o processamento de orações relativas de objeto estruturalmente não ambíguas por parte de brasileiros aprendizes tardios de inglês como L2. Optamos por essas estruturas pela simples razão de permitirem, em inglês, o uso opcional do pronome relativo that. Vejamos as sentenças de 1 - 4 a seguir:

(01)The table that Adam has in his office is old;

3 Neste artigo não estamos diferenciando segunda língua (L2) de língua estrangeira (LE). 
(02) The table $\emptyset$ Adam has in his office is old;

(03) A mesa que Adam tem em seu escritório é velha;

(04) *A mesa $\emptyset$ Adam tem em seu escritório é velha.

$\mathrm{Na}$ língua inglesa, o pronome relativo that pode ser opcionalmente usado. No português do Brasil, entretanto, isso não ocorre, pois apenas a oração com o pronome relativo "que" explícito é licenciada, como demonstrado nas frases (3) e (4) acima. Dessa forma, no inglês, o pronome relativo nulo é completamente gramatical, todavia, para a língua portuguesa a ausência do pronome gera agramaticalidade.

Seguindo esse raciocínio, como procederiam então os falantes de português como L1 processando input na L2 inglês? Essa, com certeza, é a grande questão que nos impulsiona a investigar, dentre várias hipóteses, uma possível explicação para a questão do processamento de línguas que são divergentes em relação a um determinado fenômeno linguístico.

\section{CONTEXTO TEÓRICO}

Dentre os estudos que investigam um possível processo de transferência de estratégias de processamento da L1 para a L2, destacamos Frenck-Mestre e Pynte (1997), que desenvolveram um estudo com a utilização da técnica online de rastreamento ocular (eyetracking). Nesse estudo, os autores tentam demonstrar que preferências de aposição em L2 parecem sofrer alguma influência das estratégias inicialmente empregadas na L1.

Frenck-Mestre e Pynte (1997) observaram como falantes nativos de espanhol e de inglês se comportam na leitura de orações relativas ambíguas em sua L2 (francês). Vejamos os exemplos abaixo:

(05) Jean connaîte les filles de la guardienne /qui partent.

João conhece as garotas da babá /que estão saindo (Aposição alta) ${ }^{4}$

4 Aposição alta ou ligação alta ocorre quando o sintagma mais alto ou mais distante da oração relativa está sendo referido pela oração relativa; no exemplo (05), vemos que a oração relativa se refere ao sintagma mais alto "garotas" e não ao mais baixo "babá".

Organon, Porto Alegre, nº51, julho-dezembro, 2011, p. 129-144 
(06) Jean connaîte la guardienne des filles /qui partent.

João conhece a babá das garotas que estão saindo. (Aposição baixa)

Antes de tudo, é importante observar que a preferência de resolução da ambiguidade por falantes nativos do inglês é ligar a oração relativa (doravante, $\mathrm{OR}$ ) ao sintagma nominal (doravante, $\mathrm{SN}$ ) mais próximo, enquanto no espanhol a preferência dos falantes é ligar a OR ao $\mathrm{SN}$ mais distante, semelhante ao que ocorre no francês. Quando espanhóis aprendizes de francês como L2 processavam sentenças como em (06), eles acabavam fixando o olhar por um período de tempo maior sobre o verbo, demonstrando que a estratégia de aposição que a frase impunha via concordância de número, diferentemente do espanhol, causava um estranhamento e um maior dispêndio de tempo no momento do processamento.

O mesmo efeito foi capturado para os falantes de inglês como L1 que leram frases do tipo (05) acima. Como os falantes de língua inglesa têm demonstrado uma preferência de aposição baixa e na frase a ligação da oração relativa com o sintagma mais alto está sendo forçada, uma diferença de estratégia causou uma maior fixação do olhar do aprendiz no verbo, possivelmente indicando um processo de reanálise no momento do processamento on-line.

Por outro lado, quando os falantes de ambos os grupos eram expostos às frases em que a aposição coincidia com a preferência de sua língua materna, nenhum aumento era apresentado nas medidas de tempo (milésimos de segundo) capturadas pelo aparelho de rastreamento ocular. Esse resultado foi tomado pelos autores como evidência de que, ao menos para essa estrutura, parece haver transferência de L1 para L2.

Outra hipótese bastante difundida que iremos ilustrar é a Hipótese da Estrutura Rasa (Shallow Structure Hypothesis) (Felser et al. 2002), segundo a qual aprendizes de L2 dispõem de uma gramática superficial capaz de processar com sucesso apenas estruturas de pouca complexidade e sem ambiguidades. Tal hipótese se fundamenta na idéia de que aprendizes de L2 parecem se apoiar em dicas léxico-semânticas no momento do processamento sintático. Isso ocorreria justamente porque eles não seriam sensíveis a algumas estruturas da mesma forma que um falante nativo o é. 
Estudos que focalizam tanto o processamento sintático (Felser et al. 2002) quanto o processamento morfológico (Clahsen et al. 2010) têm mostrado que aprendizes de L2, mesmo com nível de proficiência avançado, ao processarem determinados estímulos linguísticos são menos sensíveis à estrutura morfológica no caso de processamento de palavras do que nativos, assim como são menos sensíveis à informação sintática, privilegiando acesso a informação de natureza lexical.

No estudo realizado por Felser et al. (2002), também focalizando as orações relativas ambíguas, utilizando a técnica de leitura automonitorada, foi investigada a preferência de aposição entre grupos de participantes nativos gregos e alemães aprendizes de inglês como L2. É importante ressaltarmos que tanto a língua grega quanto a língua alemã, em estudos anteriores, têm demonstrado preferências de aposição alta para sentenças ambíguas, opostamente à língua inglesa, que possui preferência natural de aposição baixa à relativa.

Nesse estudo, os autores lançam mão de duas técnicas experimentais, a off-line (questionário) e a online (leitura automonitorada). No primeiro experimento, são propostas frases com diferentes condições, como as que veremos abaixo em negrito:

07) The dean liked the secretary of the professors who was reading a letter; 5

08) The dean liked the secretary of the professors who were reading a letter; 6

09) The dean liked the professors with the secretary who were reading a letter; ${ }^{7}$

10) The dean liked the professors with the secretary who was reading a letter. ${ }^{8}$

Semelhantemente, tanto para o experimento off-line quanto para o online não foram encontradas quaisquer divergências significativas na condição de genitivo complexo “of” para os aprendizes, ou seja, mesmo manipulando a ligação da oração relativa (número) aos sintagmas anteriores, não houve diferença nos tempos de processamento. Entretanto,

5 O gerente gostou da secretária dos professores que estava lendo uma carta

6 O gerente gostou da secretária dos professores que estavam lendo uma carta

7 O gerente gostou dos professores com a secretária que estavam lendo uma carta

8 O gerente gostou dos professores com a secretária que estava lendo uma carta

Organon, Porto Alegre, ํำ 51, julho-dezembro, 2011, p. 129-144 
na condição em que a preposição temática "with" ocorria, verificou-se uma maior agilidade de processamento na aposição baixa, como ilustrado na sentença (10) acima.

Esses resultados, quando comparados aos obtidos pelo grupo controle de falantes nativos de inglês, demonstram algumas semelhanças e diferenças. Nas frases em que a ligação dos sintagmas com a oração relativa era intermediada pela preposição "of" $(07,08)$ os aprendizes não demonstravam qualquer preferência de aposição definida, diferentemente dos nativos, que demonstraram uma preferência bastante clara pela aposição baixa na condição "of", como ilustrado na sentença (08). No que se refere à condição contendo "with", por outro lado, os nativos demonstraram a mesma preferência de aposição baixa que os aprendizes de L2.

Com base nesses resultados, Felser et al. (2002) defendem que não parece haver transferência das estratégias de L1 para a L2, pois, segundo eles, os aprendizes se apóiam em dicas léxico-semânticas. A justificativa para os resultados coincidentes entre nativos e aprendizes está na preposição temática with. Tal termo teria um peso informacional maior do que a preposição of. Essa informação extra forneceria dicas ou facilitaria o processamento lingüístico do aprendiz, já que ele se guia não pela estrutura sintática e sim por dicas léxico-semânticas, devido principalmente a sua gramática mais compacta.

$\mathrm{O}$ argumento utilizado é interessante, pois se somente a transferência de L1 para L2 fosse a resposta, os aprendizes deveriam ter demonstrado resultados semelhantes aos dos nativos e não resultados distintos, já que o fenômeno é idêntico nas duas línguas.

Veremos agora a descrição e análise do experimento feito com falantes nativos de português, aprendizes de inglês como L2, buscando indícios de como se dá o processamento sintático em estruturas relativas não ambíguas de objeto na L2.

\section{EXPERIMENTO}

Este experimento teve o objetivo de investigar como ocorre o processamento de orações relativas de objeto por brasileiros aprendizes de inglês como L2, com base no tempo de leitura de segmentos de frases com a presença ou a ausência do pronome that. Para tal, utilizamos sentenças comumente empregadas em língua inglesa.

As variáveis independentes foram:

Organon, Porto Alegre, nº 51, julho-dezembro, 2011,p. 129-144 
a) O nível de proficiência dos participantes, que foram divididos em: básico, intermediário e avançado;

b) Estruturas relativas de objeto em inglês, com o pronome relativo that (CT) e estruturas sem o pronome that (ST).

As variáveis dependentes foram:

a) o tempo de leitura do segmento crítico e do segmento pós-crítico;

b) o tipo de resposta à pergunta controle das sentenças.

Com essa estrutura experimental, observamos se esses aprendizes teriam mais facilidade de processar sentenças na condição CT (com that) ou se na condição ST (sem that), como nos exemplos a seguir:
$\begin{array}{lllllll}1 & 2 & 3 & 4 & 5 & 6 & 7\end{array}$
11) (CT) The/drink/ that/Mike/buys /at the beach /is delicious, ${ }^{9}$
$\begin{array}{llllll}1 & 2 & 3 & 4 & 5 & 6\end{array}$
12) (ST) The/ drink/ Mike /buys /at the beach /is delicious; ${ }^{10}$

As hipóteses aqui testadas foram:

a) Os aprendizes de L2, de maneira geral, processariam mais facilmente as estruturas que se assemelhassem à sua L1, ou seja, sentenças contendo that, semelhante ao que ocorre no português;

b) Os tempos de leitura mais lentos seriam dos aprendizes com nível de proficiência básico e os mais rápidos seriam dos aprendizes com nível de proficiência avançado, mostrando uma influência direta do nível de proficiência na velocidade de processamento;

c) O processamento dos aprendizes seria diretamente afetado pelo nível de proficiência. Sendo assim, os aprendizes de nível básico teriam mais dificuldades de processar as estruturas na L2 e, especificamente, na condição ST que se distancia da L1, do que os aprendizes de nível intermediário e avançado.

\subsection{Método}

Participantes

Foram voluntários desse experimento 24 participantes falantes nativos do português brasileiro, aprendizes tardios ${ }^{11}$ de inglês como língua

\footnotetext{
$9 \quad$ A bebida que Mike compra na praia é deliciosa.

10 A bebida que Mike compra na praia é deliciosa.

11 Consideramos os participantes aprendizes tardios de inglês por todos terem começado a estu-

Organon, Porto Alegre, nº 51, julho-dezembro, 2011, p. 129-144
} 
estrangeira, em sua maioria alunos do curso de graduação de Língua Inglesa. Os demais participantes foram alunos de um curso de idiomas de João Pessoa como também alunos de pós-graduação. Dentre esses participantes, tivemos 14 do sexo masculino e 10 do sexo feminino com idade média de 26 anos.

Material

O material consistia em 2 conjuntos de 10 frases experimentais para cada condição. Cada participante era exposto a um desses conjuntos experimentais, embutidos em um pacote extra de 24 frases distratoras.

As frases experimentais foram formadas por orações relativas restritivas não ambíguas, todas sendo afirmativas e estando no tempo presente. Tais sentenças foram subdivididas em 6 ou 7 seg$\operatorname{mentos}^{12}$, a depender da condição, sendo que o segmento de número 4 seria o segmento crítico analisado na condição CT, ficando o segmento 5 em observação em caso ocorrência de efeito spillover. ${ }^{13}$ Para a condição ST, o segmento crítico foi o de número 3 e o segmento 4 o pós-crítico, justamente pela subtração do pronome relativo that das sentenças.

As frases foram formadas por orações relativas nas condições CT e ST e tiveram para cada condição separadamente o mesmo número de segmentos, sendo o tamanho das palavras dentro das sentenças rigorosamente controlado para que o experimento se fizesse homogêneo.

Para combinarmos as sequências de frases que seriam lidas, utilizamos o quadrado latino ${ }^{14}$ com a intenção de que cada participante tivesse contato com todos os tipos de estruturas, sem que houvesse repetição de sentenças no momento da leitura.

Lembramos que, ao final da leitura de cada sentença, sempre havia uma pergunta controle ${ }^{15}$ que focalizava o conteúdo expresso na sen-

dar a língua estrangeira depois da puberdade.

12 As frases experimentais encontram-se em anexo.

13 Spillover - ocorre quando um efeito esperado para um segmento (x) só se expressa no segmento (y) seguinte.

14 Quadrado latino (latin square) é uma matriz matemática ( $\mathrm{n} \times \mathrm{n}$ ) de mesmo número de colunas e linhas que permite que os estímulos sejam distribuídos de tal forma que cada sujeito é exposto a todas as condições experimentais sem repetir o estímulo, o que evita efeitos indesejáveis de repetição e reativação.

15 Exemplo de pergunta controle em relação aos exemplos (11) e (12): Is the drink delicious? 
tença. Depois de ler a pergunta, o participante respondia com sim ou não pressionando um botão do teclado. O intuito principal da pergunta controle era verificar se o número de acertos e o tempo de resposta final variariam entre os níveis de proficiência dos participantes. Com a pergunta controle, ainda teríamos a possibilidade de identificar casos em que os voluntários estivessem respondendo ao experimento aleatoriamente, prejudicando assim os resultados finais.

$\mathrm{O}$ aparato experimental consistiu do uso de um Mac book 13 $(2,4 \mathrm{GHz})$, pertencente ao LAPROL-UFPB ${ }^{16}$, para a utilização com o programa Psyscope, que permite a aferição da leitura em milissegundos (COHEN, J. D., MACWHINNEY, B., FLATT, M., \& PROVOST, S., 1993).

\section{Procedimento}

O experimento consistia basicamente de duas etapas. Como os níveis de proficiência são condições de uma das variáveis independentes testadas, fez-se necessário controlar o grau de conhecimento que cada participante tinha da L2 inglês.

Para essa tarefa, adotamos o teste de nivelamento da Oxford Placement Test, de Dave Allen, de $2004^{17}$, que foi aplicado por completo para todos os participantes. O teste consistia de 200 questões objetivas, sendo que 100 questões eram de gramática/compreensão (Grammar test) e 100 questões de audição (listening test) em língua inglesa. A aplicação do teste de nivelamento foi feita em uma sala tranquila e isolada de barulho. Os participantes gastaram um tempo médio de 40 minutos para responderem todas as questões.

Depois de concluída a etapa de aplicação dos testes de proficiência e feita a classificação de cada voluntário pelos níveis de proficiência ${ }^{18}$, pudemos partir para a etapa seguinte de aplicação efetiva do experimento de leitura automonitorada (Self Paced Reading).

16 LAPROL - Laboratório de Processamento Linguístico da UFPB. Site: http://www.cchla.ufpb. $\mathrm{br} / \mathrm{laprol} /$

17 Precisamos registrar que toda a parte de aplicação do teste de nivelamento teve a supervisão do professor Rubens Marques de Lucena, do Departamento de Letras Estrangeiras Modernas da UFPB. Aqui fica o nosso agradecimento.

18 Os participantes que obtiveram pontuação de 0 a 104 pontos foram considerados do nível básico de proficiência, os que obtiveram de 105 a 134 pontos foram considerados do nível intermediário e os que obtiveram de 135 pontos para cima foram considerados de nível avançado.

Organon, Porto Alegre, nº 51, julho-dezembro, 2011, p. 129-144 
Os participantes eram postos sentados em frente à tela do computador (Mac book) e eram oralmente orientados pelo experimentador sobre os comandos e procedimentos do experimento. Logo em seguida, realizava-se uma prática, com frases semelhantes às experimentais, para que o participante pudesse manusear e se familiarizar com o equipamento.

O início da tarefa propriamente dita consistia na leitura de uma sentença fragmentada em 6 ou 7 segmentos. O participante lia as instruções e, ao clicar no botão previamente indicado do teclado, o experimento iniciava com o surgimento do primeiro segmento. Para que o participante pudesse ler toda a frase, era necessário que ele pressionasse o botão a cada segmento lido, assim o segmento anterior sumiria da tela e o seguinte surgiria. Ao final da leitura de todos os segmentos e consequentemente da sentença, uma pergunta surgia na tela do computador e o voluntário só precisava responder teclando sim ou não nos botões indicados.

A média de tempo gasto para aplicação do experimento foi de 15 minutos, levando-se em consideração as orientações dadas, a prática realizada e a aplicação efetiva do experimento.

\section{RESULTADOS E DISCUSSÃO}

Os resultados do experimento relacionados ao tempo de leitura do segmento crítico, descritos na Figura 1, a seguir, mostram que houve um efeito principal do nível de proficiência dos participantes (ANOVA: $\mathrm{F}(2,21)=5,93, \mathrm{p}<0,009)$, sendo que o grupo com nível de proficiência avançado foi mais rápido em comparação com os grupos com nível de proficiência básico e intermediário, tanto na condição sem o that (ST), quanto na condição com that (CT). Houve também um efeito principal do tipo de estrutura (ANOVA: $\mathrm{F}(1.21)=10,1, \mathrm{p}<0,004$ ); de maneira geral, a condição com that (CT) foi lida mais rapidamente do que a condição sem o that (ST). Houve ainda um efeito marginal de interação (ANOVA: $\mathrm{F}(2,21)=2,86, \mathrm{p}<0,07)$, e somente no nível básico e intermediário houve diferença significativa entre CT e ST, o que é corroborado pelos testes t pareados: Test-t básico (CT x ST): $\mathrm{t}(14)=3,64, \mathrm{p}<0,008)$, Test-t Intermediário (CTxST): $\mathrm{t}(14)=2,20, \mathrm{p}<0,05)$, Test- $\mathrm{t}$ avançado $(\mathrm{CT} \mathrm{x} \mathrm{ST}): \mathrm{t}(14)=0,12, \mathrm{p}=0,9)$.

Organon, Porto Alegre, nº 51, julho-dezembro, 2011, p. 129-144 


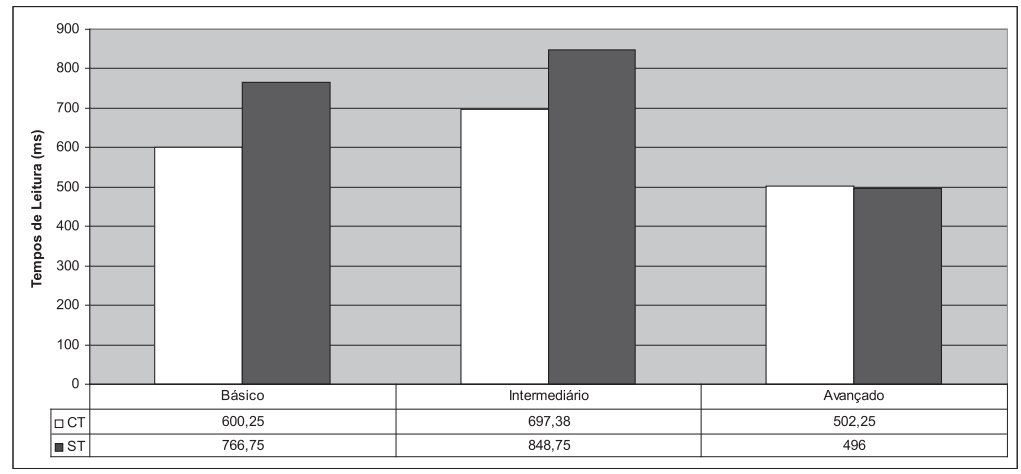

Fig. 1 - Médias de tempo de leitura do segmento crítico por condição

Os resultados explicitados na Figura 1, referentes aos tempos de leitura do segmento crítico, vão ao encontro de nossas hipóteses, por mostrarem que realmente o nível de proficiência parece ter um papel no processamento linguístico, pois o grupo avançado processa significativamente mais rápido do que os outros dois grupos. Além disso, nos níveis básico e intermediário, encontramos um efeito significativo do tipo de estrutura, dado que o segmento crítico da estrutura com that (CT) na L2, semelhante à estrutura com "que" licenciada em português, foi processado mais rapidamente do que o segmento crítico da estrutura sem o that (ST), que é possível apenas na L2. Isso pode ser indício de que haja transferência de L1 para L2 no processamento desse tipo de estrutura.

Apresentamos, na Figura 2 a seguir, os resultados referentes aos tempos de leitura no segmento pós-crítico, para verificarmos um possível efeito de spillover. Os resultados mostram que houve de novo um efeito principal do nível de proficiência (ANOVA: $\mathrm{F}(2,21)$ $=8,75, \mathrm{p}<0,001$ ), sendo que mais uma vez o grupo avançado foi mais rápido na leitura do que os grupos de nível básico e intermediário. Houve também um efeito principal relacionado ao tipo de estrutura (ANOVA: $\mathrm{F}(1,21)=1,20, \mathrm{p}<0,03$ ), já que os tempos foram menores para a estrutura do tipo CT do que para a estrutura do tipo ST em todos os níveis de proficiência. Entretanto, observando mais uma vez os testes-t pareados: Test-t básico (CT x ST): $\mathrm{t}(14)=0,59$, $\mathrm{p}=0,5)$, Test-t intermediário (CT x ST): $\mathrm{t}(14)=1,77, \mathrm{p}=0,1) \mathrm{e}$ Organon, Porto Alegre, nº 51, julho-dezembro, 2011, p. 129-144 
Test-t avançado (CT x ST): $\mathrm{t}(14)=2,26, \mathrm{p}<0,05$, verificamos que só se mostrou significativa a diferença entre CT e ST no grupo avançado, ou seja, houve um efeito de spillover capturado mais claramente no nível avançado.

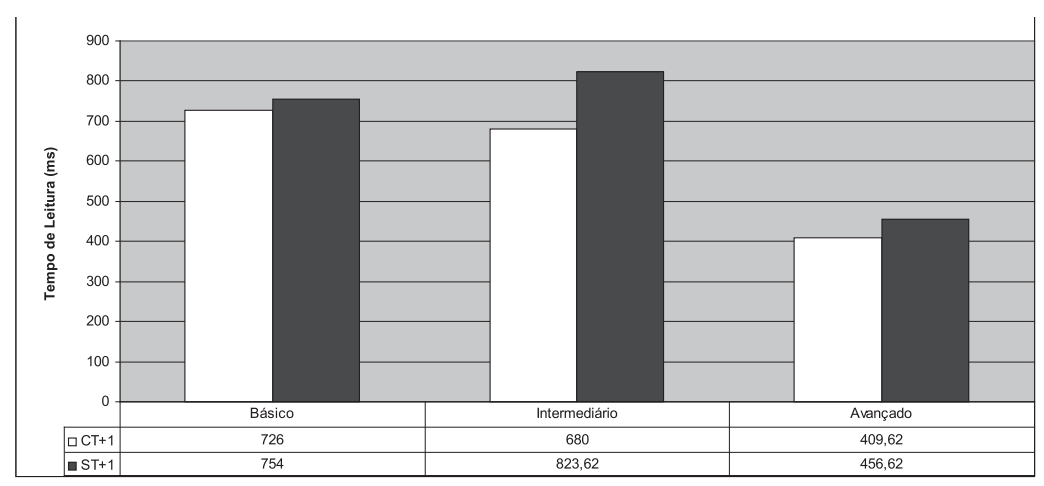

Fig. 2 - Média dos tempos de leitura dos segmentos pós-críticos por condição

Esses resultados, referentes ao segmento após o segmento crítico, são interessantes por mostrarem que, apesar de os participantes de nível avançado continuarem lendo bem mais rapidamente, agora há um efeito do tipo de estrutura também no nível avançado na direção dos resultados encontrados para o segmento crítico nos níveis básico e intermediário, pois a condição CT foi lida mais prontamente do que a condição ST. Isso indica que também no grupo avançado teríamos uma possível transferência de L1 para L2 no processamento desse tipo de construção sintática. Talvez o fato de termos encontrado esse efeito no segmento seguinte seja um indicativo de que essa transferência em grupos com uma proficiência avançada pode se manifestar posteriormente em termos de processamento sintático.

Após a leitura de cada frase, os participantes eram expostos às perguntas controle, que tinham a finalidade de verificar se os tempos de respostas variariam de acordo com os diferentes níveis de proficiência dos participantes; elas também tinham o objetivo de fixar a atenção do participante ao experimento e ajudar o experimentador a identificar possíveis candidatos que não estivessem atentos ao momento de experimentação. Podemos observar os tempos de resposta à pergunta de final de frase, a partir da Figura 3, a seguir:

Organon, Porto Alegre, nº 51, julho-dezembro, 2011,p. 129-144 


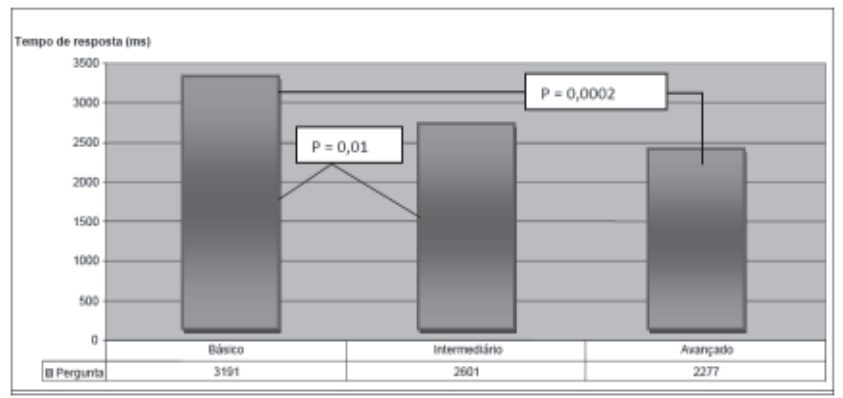

Fig. 3. Médias do tempos de respostas por nível de proficiência

Lembramos que o percentual de acertos das perguntas controle pelos participantes do experimento foi superior a 90\%, confirmando que os voluntários desta pesquisa realmente estavam atentos às questões propostas e conseguiram chegar a uma compreensão satisfatória em relação ao conteúdo das sentenças.

Os resultados do grupo básico mostram que os participantes gastaram mais tempo para ler e responder à pergunta final, 3.190 milésimos de segundo em média, do que os grupos intermediário e avançado.

Os tempos de resposta do grupo de nível intermediário foram significativamente mais rápidos que o grupo básico, tanto para leitura quanto para as respostas às perguntas controle. Comparando-se os tempos do grupo básico e do grupo intermediário, obtivemos diferença estatisticamente significativa nos tempos de resposta, indicando que os participantes de nível intermediário responderam às perguntas controle mais rapidamente do que os de nível básico (Test-t: $\mathrm{t}(14)=2,10, \mathrm{p}=0,018$ ).

Comparando-se os tempos de resposta à pergunta controle entre o grupo básico e o grupo avançado, obtivemos também uma diferença estatística relevante nos tempos de resposta (Test-t $(176)=3,54, \mathrm{p}=0,0002$ ). Sendo assim, o grupo com nível de proficiência avançado demonstrou ser muito mais veloz para a leitura da sentença e também para o tempo de resposta à pergunta do que os grupos básico e intermediário.

Os resultados referentes aos tempos de resposta à pergunta final são interessantes porque reforçam a influência do nível de proficiência no processamento, seja em tarefas on-line, como a leitura automonitorada, seja em tarefas off-line, como as repostas de final de frase. Esses resulta- 
dos também corroboram o resultado do teste de proficiência aplicado, mostrando que os níveis apontados para os participantes parecem ser compatíveis com o desempenho deles em termos de processamento.

As análises nos mostram que há indício de que tenha ocorrido transferência de L1 para L2 no tipo de estrutura testado, no entanto não podemos descartar a possibilidade explicativa da Shallow Structure, já que os participantes dos três níveis de proficiência demonstraram um processamento mais rápido com a estrutura CT, a qual é semelhante à L1 e tem a dica lexical do pronome that presente, evidenciando uma possível distinção em relação ao processamento sintático entre sujeitos que adquiriram a L2 mais cedo ou mesmo concomitantemente e sujeitos, como os do nosso experimento, que aprenderam tardiamente a L2 e que mesmo com um nível avançado de proficiência mostraram um possível efeito de transferência.

É importante ressaltarmos que precisaríamos coletar dados com um grupo controle de falantes nativos de inglês, para verificarmos se eles seriam mais lentos no processamento na condição ST, em detrimento da condição CT, como ocorreu com os aprendizes. Se forem, poderemos afirmar que as sentenças sem o that seriam mais complexas e realmente mais difíceis de processar, por falta da dica lexical do pronome relativo. Já se os tempos de processamento nos falantes nativos de inglês forem semelhantes para ambas as condições, isso reforçará a evidência de que houve transferência de L1 para L2 no processamento de relativas não ambíguas.

\section{CONSIDERAÇÕES FINAIS}

Com base nos resultados obtidos neste estudo, identificamos que o nível de proficiência dos aprendizes parece realmente ser um fator relevante para que o processamento se dê de forma mais ou menos ágil. Como foi demonstrado nos resultados, verificamos que os tempos de processamento dos três grupos são proporcionalmente menores à medida que o nível de proficiência se eleva. Isso indica que quanto maior o nível de proficiência maior a habilidade de processamento na L2.

Observamos que os aprendizes de maneira geral demonstraram ler mais rapidamente estruturas que se aproximam das utilizadas em suas línguas maternas. Isso talvez ocorra porque as sentenças na L2 seguem 
as mesmas estruturas do português, demonstrando um efeito de transferência. No entanto, só poderemos afirmar isso de forma mais categórica quando obtivermos resultados de um grupo controle formado por falantes nativos de inglês.

Podemos concluir que a Shallow Structure Hypothesis e a Hipótese da Transferência são, até certo ponto, complementares e explicam satisfatoriamente a dificuldade apresentada pelos aprendizes de L2 no momento de processamento. Contudo, ainda precisamos de mais pesquisas com grupos controles para averiguar se esse efeito se reflete também em tal público ou não.

Nosso estudo parece, então, ser significativo ao abrir caminho para que outros experimentos e outras análises sejam feitos, no intuito de corroborarem ou não as hipóteses existentes na literatura e complementarem os resultados explicitados aqui.

\section{ANEXO}

\section{Conjunto de frases experimentais e perguntas controle} Com That (CT)

1a - The / game / that /Fred / plays/ at night/ is difficult. $2 \mathrm{a}$ - The / book / that / Mary / shows / to the class/ is excellent. 3a - The/ drink/ that /Mike/buys /at the beach /is delicious.

$4 \mathrm{a}$ - The / snake /that /John / kills/ in his house/ is dangerous. $5 \mathrm{a}-$ The / ball/ that /Luke / uses / on the game/ is good. $6 \mathrm{a}$ - The / man/ that /Lisa / loves/ at the city/ is young. $7 \mathrm{a}$-The /song/ that /Paul / sings/ in the show / is beautiful. $8 \mathrm{a}$ - The / table/ that / Adam / has/ in his office/ is old; 9a - The / food / that /Kate /eats / at the market/ is gratuitous; 10a -The /aquarium /that /Mike/ wants /to his fish/ is big.

Sem That (ST)

$1 \mathrm{~b}$ - The / game /Fred / plays/ at night/ is difficult; $2 \mathrm{~b}$ - The / book / Mary / shows / to the class/ is excellent; $3 \mathrm{~b}$ - The/ drink /Mike /buys /at the beach /is delicious; $4 \mathrm{~b}$ - The / snake /John / kills/ in his house/ is dangerous; $5 \mathrm{~b}$ - The / ball /Luke / uses / on the game/ is good;

Organon, Porto Alegre, nº 51, julho-dezembro, 2011, p. 129-144 
$6 \mathrm{~b}$ - The / man /Lisa / loves/ at the city/ is young;

$7 \mathrm{~b}$ - The /song /Paul / sings/ in the show / is beautiful;

$8 \mathrm{~b}$ - The / table/ Adam / has/ in his office/ is old;

$9 \mathrm{~b}$ - The / food /Kate /eats / at the market/ is gratuitous;

$10 \mathrm{~b}$ - The /aquarium /Mike/ wants /to his fish/ is big.

\section{BIBLIOGRAFIA}

ALLEN, David. The Oxford Placement Test. Oxford: Oxford University Press, 2004.

CLAHSEN, H.; FELSER, C.; NEUBAUER, K.; SATO, M.; SILVA, R. Morphological structure in native and nonnative language processing. Language Learning. 60:1, 21- 43. 2010. COHEN, J. D.; MacWHINNEY, B.; FLATT, M.; PROVOST, S. Psyscope: a new graphic interactive environment for designing psychology experiments. Behavioral Research Methods, Instruments \& Computers. 25(2), 257-271. 1993.

FELSER, C. et al. The processing of ambiguous sentences by first and second language learners of English. Essex Research Reports in Linguistics, Colchester, v.40, p.1-38, 2002.

FELSER, C.; CLAHSEN, H. Grammatical processing of spoken language in child and adult language learners. Journal of Psycholinguistic Research, 38, 305 - 309. 2009.

FRENCK-MESTRE, C.; PYNTE, J. Syntactic ambiguity resolution while reading in second and native languages. Quarterly Journal of Experimental Psychology, v. 50A, p. 119-148, 1997. 